\title{
Bilateral multifocal synchronous intra- and extra-parotid Warthin tumors: case report and review of literature
}

\author{
Beatrice FABIANO, Giuseppe RIVA*, Vittorio FERRERO \\ ENT Department, Gradenigo Hospital, Turin, Italy
}

*Corresponding author: Giuseppe Riva, ENT Department, Gradenigo Hospital, Corso Regina Margherita 10, 10153 Turin, Italy. E-mail: giuseppe.riva84@gmail.com

\section{A B S T R A C T}

Warthin tumor is the second most frequent benign tumor of the parotid gland. Multifocal Warthin tumors are rarely bilateral synchronous. Six synchronous bilateral cases with more than two nodules of Warthin tumor have been described in the literature; only three cases had 4 or 5 tumor nodules. None of these had also extra-parotid localizations. We report a rare case of a patient with four bilateral synchronous Warthin tumors of parotid glands and we present a complete review of the literature. A 72-year-old woman presented at our division with a 12 months history of bilateral parotid swelling. Magnetic resonance imaging revealed four bilateral nodular lesions of the parotid glands. Fine-needle aspiration biopsy was indicative of Warthin tumors. The patient underwent surgical treatment. Multifocality should be kept in mind when a Warthin tumor is diagnosed. Therefore, magnetic resonance imaging represent an important tool in the diagnostic algorithm, and an adequate follow-up is mandatory.

(Cite this article as: Fabiano B, Riva G, Ferrero V. Bilateral multifocal synchronous intra- and extra-parotid Warthin tumors: case report and review of literature. Otorinolaringol 2017;67:120-2. DOI: 10.23736/S0392-6621.17.02123-3)

Key words: Adenolymphoma - Parotid gland - Salivary gland neoplasms.

$\mathrm{W}$ arthin tumor, also known as papillary cystadenoma lymphomatosum, is the second most frequent benign tumor of the parotid gland after pleomorphic adenoma. ${ }^{1}$ The tumor accounts for $2-15 \%$ of all parotid tumors. ${ }^{2,3}$

Salivary gland neoplasms are usually solitary while multiple tumors occurring simultaneously in an individual are uncommon. Most of the multifocal Warthin tumors are unilateral, whereas bilateral Warthin tumors are much more uncommon. Most of the bilateral tumors are metachronous, with a very few cases of synchronous bilateral tumors reported in the literature. ${ }^{2,4-7}$ Only one previous case had simultaneous bilateral parotid and neck localizations. ${ }^{5}$

We present a rare case of a patient with four bilateral synchronous intra- and extra-parotid Warthin tumors. Written informed consent was obtained.

\section{Case report}

A 72-year-old woman presented at our division with a 12 months history of bilateral parotid swelling. Apart from the habit of smoking, her medical history was normal. Clinical examinations confirmed bilateral mobile, painless swelling, without intra-oral lesions. Facial nerves function was normal. Ultrasonography (US) showed multifocal bilateral parotid nodules, so magnetic resonance imaging (MRI) was programmed.

MRI revealed four bilateral nodular lesions of the parotid glands: three nodules in the right parotid gland and one nodule in the left gland. They were well defined and rounded in appearance, with mild enhancement after administration of intravenous contrast medium (Figure 1). The left nodule measured $2 \mathrm{~cm}$ in diameter and it was in the superficial lobe. In the right parotid gland, the bigger nodules measured $2.2 \mathrm{~cm}$ and $2.1 \mathrm{~cm}$ in maximum dimension, while a minor nodule had a $1 \mathrm{~cm}$ diameter. The $2.1 \mathrm{~cm}$ nodule was in the deep lobe of right parotid gland.

Fine-needle aspiration biopsy (FNAB), under ultrasonography guidance, was carried out on the bigger right nodule and on the left nodule. It was indicative of Warthin tumors. 


\section{COPYRIGHT ${ }^{\odot} 2017$ EDIZIONI MINERVA MEDICA}

FABIANO

WARTHIN TUMORS

grade lymphoma is recognized. Moreover, an extra-parotid neck localization can mimic a tumor metastatic to a lymph node.

Diagnosis is based on histological examination, so ultrasound-guided fine needle aspiration cytology or biopsy (FNAC or FNAB) is mandatory in the diagnostic algorithm. Moreover, in multifocal tumors, complete bilateral screening of the parotid glands by MRI is needed to program surgery.

The treatment recommended for Warthin tumor is superficial parotidectomy, when the tumor occurs in a superficial lobe, or total parotidectomy, when the tumor is in a deep lobe, with preservation of the facial nerve. The recurrence rate is low, most likely due to multifocality. ${ }^{1}$ For this reason, a meticulous macroscopic examination of the parotid is indicated. Moreover, a meticulous intra-operative inspection and palpation of periglandular lymph nodes is mandatory in order to exclude the presence of extra-parotid Warthin tumors. Our patient underwent right total parotidectomy, in order to first treat the parotid gland with the majority of tumor nodules. The patient have chosen a waiting and see approach for the left Warthin tumor. Therefore, it is mandatory to discuss the therapeutic path with the patient.

Long-term follow-up is mandatory, due to the possible occurrence of metachronus salivary and extrasalivary tumors, even after prolonged time intervals. US should be used in follow-up. Concerning multifocal bilateral Warthin tumors, literature studies report a disease free follow-up between 3 months and 5 years. ${ }^{4}, 10$ No recurrence on the operated side was observed at 6 months after surgery in our case. ${ }^{10}$

\section{Conclusions}

Multiple Warthin tumors, synchronously involving both parotid glands, are exceptionally rare. However, multifocality and extra-parotid localizations should be kept in mind when a Warthin tumor is diagnosed. Therefore, MRI examination represents an important tool in the diagnostic algorithm, an intra-operative macroscopic examination of the parotid is indicated, and an adequate follow-up is mandatory.

\section{References}

1. Simpson RHW, Eveson JW. Warthin tumor. In: Barnes L, Eveson JW, Reichart P, Sidransky D (ed) World Health Organization classification of tumors: pathology and genetics of head and neck tumors. Lyon, France: IARC Press, 2005:263-5.

2. Maiorano E, Lo Muzio L, Favia G, Piattelli A. Warthin's tumor: a study of 78 cases with emphasis on bilaterality, multifocality and association with other malignancies. Oral Oncology 2002;38:35-40

3. Hilton JM, Phillips JS, Hellquist HB, Premachandra DJ. Multifocal multi-site Warthin tumor. Eur Arch Otorhinolaryngol. 2008;265:1573-5

4. Ascani G, Pieramici T, Rubini C, Messi M, Balercia P. Synchronous bilateral Warthin's tumors of the parotid glands: a case report. Acta Otorhinolaryngol Ital 2010;30:310-2.

5. Shah N, Tighe JV, Barrett AW, Kumar S, Allen JP. Bilateral intraparotid and extraparotid Warthin's tumors. Br J Oral Maxillofac Surg 2007:45:238-9

6. Kremp AN, Nelson BL. Bilateral Warthin tumors of the parotid gland. Head and Neck Pathol 2008;2:175-6.

7. Nascimento LA, Santos Ferreira JA, Pio RB, Soares Takano GH, Miziara HL. Synchronous bilateral Warthin tumors: a case report. Int Arch Otorhinolaryngol 2014;18:217-20.

8. Klussmann JP, Wittekindt C, Preuss SF, Al Attab A, Schroeder U, Guntinas-Lichius O. High risk for bilateral Warthin tumor in heavy smokers - review of 185 cases. Acta Oto-Laryngologica 2006;126:1213-7.

9. Yu GY, Ma DQ, Zhang Y, Peng X, Cai ZG, Gao Y, et al. Multiple primary tumors of the parotid gland. Int J Oral Maxillofac Surg 2004;33:531-4.

10. Deveer M, Sahan M, Sivrioglu AK, Celik OI. Bilateral multifocal Warthin tumors. BMJ Case Rep 2013;2013.

Conflicts of interest.-The authors certify that there is no conflict of interest with any financial organization regarding the material discussed in the manuscript. Manuscript accepted: July 24, 2017. - Manuscript received: July 15, 2017. 